\title{
Origine et expansion de l'Afro-Asiatique : méthodologie pour une approche pluridisciplinaire
}

The origin and expansion of Afro-Asiatic: Methodology for a pluridisciplinary approach

\section{Ben Hamed et Pierre Darlu}

\section{(2) OpenEdition}

10 Journals

Édition électronique

URL : https://journals.openedition.org/bmsap/564

DOI : 10.4000/bmsap.564

ISSN : $1777-5469$

Éditeur

Société d'Anthropologie de Paris

Édition imprimée

Date de publication : 1 juin 2003

ISSN : 0037-8984

Référence électronique

M. Ben Hamed et Pierre Darlu, « Origine et expansion de l'Afro-Asiatique : méthodologie pour une approche pluridisciplinaire », Bulletins et mémoires de la Société d'Anthropologie de Paris [En ligne], 15 (1-2) | 2003, mis en ligne le 19 janvier 2021, consulté le 21 septembre 2021. URL : http:// journals.openedition.org/bmsap/564 ; DOI : https://doi.org/10.4000/bmsap.564

\section{(c) $\underset{\mathrm{EY}}{(\mathrm{NQ} \mathrm{NO}}$}

Les contenus des Bulletins et mémoires de la Société d'Anthropologie de Paris sont mis à disposition selon les termes de la licence Creative Commons Attribution-NonCommercial-NoDerivatives 4.0 International License. 


\title{
ORIGINE ET EXPANSION DE L'AFRO-ASIATIQUE : MÉTHODOLOGIE POUR UNE APPROCHE PLURIDISCIPLINAIRE
}

\author{
THE ORIGIN AND EXPANSION OF AFRO-ASIATIC: METHODOLOGY \\ FOR A PLURIDISCIPLINARY APPROACH
}

\author{
Mahé Ben Hamed ${ }^{1}$, 2 , Pierre Darlu 1
}

\section{RÉSUMÉ}

Le groupe linguistique afro-asiatique occupe un domaine géographique à cheval entre Afrique et Asie, une répartition qui pose la question de la localisation du berceau de cette famille. Linguistique, génétique des populations et archéologie se sont penchées sur cette question sans toutefois parvenir à y répondre. La spécificité des approches de chacune de ces disciplines, ainsi que leur nature interprétative, rendent la question du foyer encore plus complexe et appellent à une reformulation méthodologique de cette problématique. Cet article constitue une réflexion méthodologique sur la problématique de l'évolution d'un phylum linguistique et tente de définir un cadre théorique global pour aborder l'évolution des populations humaines de façon syncrétique, biologique et culturelle à la fois. Nous y présentons une revue critique des approches linguistiques et extralinguistiques de la question du foyer de l'Afro-Asiatique. Nous proposons une méthodologie unique tenant compte des limites observées dans les approches actuelles et passées sur cette question, de considérations propres à chacune des disciplines concurrentes ainsi que des incidences dues à la nature même des données disponibles.

Mots-clés : Afro-Asiatique, foyer originel, génétique, morphologie, linguistique, méthodologie.

\section{ABSTRACT}

The Afro-Asiatic linguistic group occupies a geographical zone which straddles two continents, Africa and Asia, a distribution which brings into question the location of this family's homeland. Linguistics, Population Genetics and Archaeology have tried unsuccessfully to provide an answer, because the specificity and the interpretative nature of their different approaches make the homeland issue even more complex, and calls for reconsideration of the methodology used. This paper is a methodological reflection on the

1. Inserm U535, Génétique Épidémiologique et Structure des Populations Humaines, Bâtiment Leriche, Secteur Jaune, $1^{\text {er }}$ étage, BP 1000, 94817 Villejuif CEDEX, France, e-mail : darlu@ vjf.inserm.fr

2. UMR 5596 du CNRS, Laboratoire de Dynamique du Langage, Institut des Sciences de l'Homme, 14 avenue Berthelot, 69363 Lyon CEDEX 07, France, e-mail : Mahe.Ben-Hamed@ish-lyon.cnrs.fr 
development of a linguistic group and an attempt to define an overall theoretical framework to consider the development of human populations in syncretic, biological and cultural terms. We present here a critical review of the linguistic and extra-linguistic methods applied to the question of an Afro-Asiatic homeland. We propose a single methodology taking into account the limitations observed in past and current works on the subject, the considerations related to each discipline as well as the particular impacts due to the nature of the available data.

Key words: Afro-Asiatic, original homeland, genetics, morphology, linguistics, methodology.

\section{LOCALISATION DU BERCEAU DE L'AFRO-ASIATIQUE : UNE PROBLÉMATIQUE OUVERTE}

L'Afro-Asiatique (Cohen 1924) est une famille linguistique actuellement subdivisée en six branches, dont une éteinte, l'Égyptien. Les cinq autres branches actuelles, très diversifiées, sont le Sémitique, le Berbère, le Couchitique, le Tchadique - depuis Greenberg (1955, 1966) - et l'Omotique (Diakonoff 1965, 1988). La distribution particulière de cette famille lui a donné son nom. Elle est à cheval sur les continents africain et asiatique et comprend un passage emprunté à plusieurs reprises et dans les deux sens (Hammer et al. 1998 ; Passarino et al. 1998 ; Cruciani et al. 2002) au cours de l'histoire du peuplement humain (fig. 1). Cette répartition pose avec acuité la question de la localisation de son foyer originel : africain ou asiatique ?

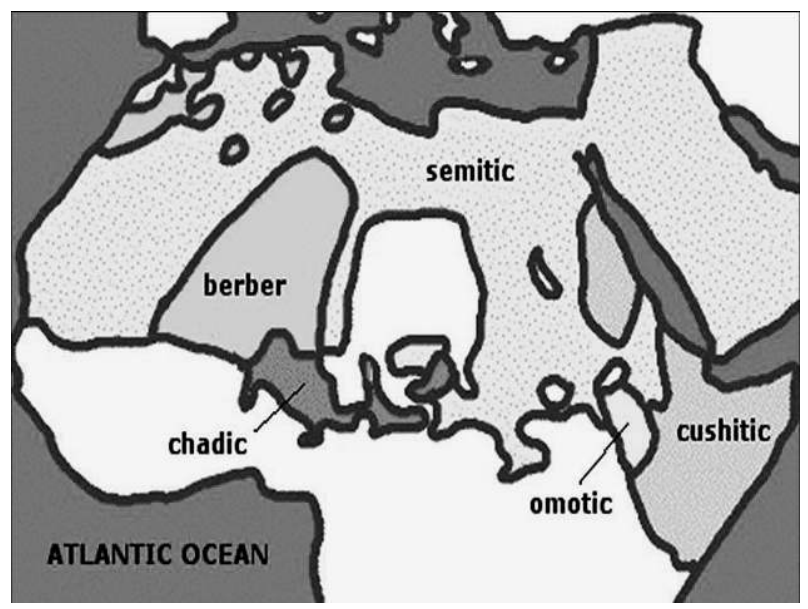

Fig. 1 - Carte géographique du domaine afro-asiatique. Fig. 1-Geographical map of the Afro-Asiatic zone. 
Cette question, contemporaine de l'intuition de parenté entre ces branches, a fait couler beaucoup d'encre, et des thèses contradictoires sont encore défendues. Entre linguistes d'abord, qui ont proposé de nombreuses classifications non concordantes pour l'Afro-Asiatique. De plus, la délimitation de certaines branches n'a toujours pas emporté le consensus. Les topologies proposées pour cette famille diffèrent dans les positions relatives du Sémitique et de l'Omotique (fig. 2). Certains auteurs estiment que le Sémitique a divergé en premier (Vicychl 1981; Militarev et al. 1988). C'est la position de l'école linguistique russe, ardent défenseur du Nostratique et anciennement sémitocentrique. Toutefois, une majorité d'auteurs considèrent que l'Omotique est la branche la plus ancienne (Blench 1995 ; Ehret 1995).

Chamito-Sémitique (Vicychl 1981)

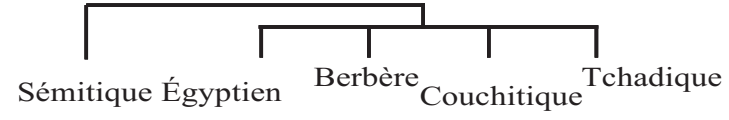

Afro-Asiatique (Ehret 1979)

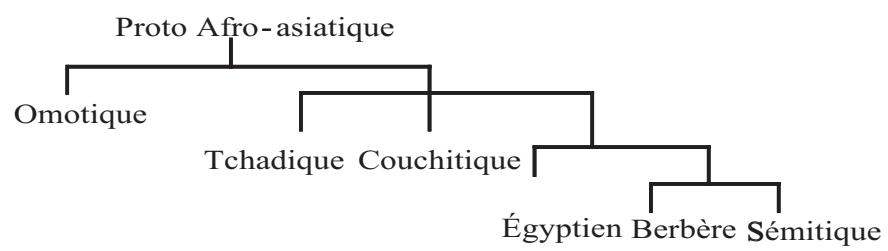

Afro-Asiatique (Blench 1994)

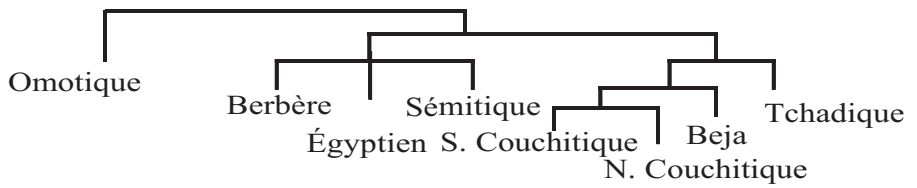

Fig. 2 - Classifications de l'Afro-Asiatique. Vicychl représente la position sémitocentrique, Ehret et Blench représentent le courant majoritaire.

Fig. 2 -Classifications of the Afro-Asitic family. Vicychl represents the semito-centric view whereas Ehret and Blench represent the main trend. 
D'autres disciplines, telles que la génétique des populations ou l'archéologie, ont apporté leur cortège de méthodes et d'arguments pour participer à un débat linguistique déjà foisonnant, sans pour autant permettre d'y apporter une réponse définitive. Plus fondamentalement, l'approche pluridisciplinaire a mis l'accent sur des faiblesses méthodologiques des arguments linguistiques en en prenant le contre-pied et en offrant un soutien inopiné à une faction minoritaire de linguistes. Elle propose de replacer la problématique du berceau de l'Afro-Asiatique dans une perspective plus large d'histoire du peuplement humain.

\section{L'argumentaire linguistique}

L'argumentaire strictement linguistique, dans son courant majoritaire, conclut de façon relativement cohérente à un berceau africain. L'hypothèse " age-area », reformulée par Sapir (1916) pour la linguistique, définit que le foyer le plus vraisemblable des premiers groupes de locuteurs d'une famille donnée est localisé là où la plus grande diversité linguistique est observée actuellement. Dans sa description de l'AfroAsiatique, Greenberg (1971) reformule cet argument sous la forme du scénario des «least-moves ». Il définit le continent africain comme l'hypothèse la plus parcimonieuse de localisation du berceau de cette famille au vu de sa distribution actuelle : quatre des cinq branches actuelles sont africaines, la branche éteinte était également de localisation africaine, et seul le Sémitique est représenté en Asie, tout en étant également représenté en Afrique. Le scénario d'expansion le plus parcimonieux impliquerait une migration du Sémitique d'Afrique en Asie, à partir d'un berceau africain, dont la localisation serait éthiopienne, lieu d'une grande hétérogénéité linguistique. Ces deux hypothèses, « age-area » et « least-moves », font appel à des principes théoriques généraux, non spécifiques à la linguistique, et qui, bien que trans-disciplinaires, sont sensibles à des particularismes.

Les inférences issues de méthodologies proprement linguistiques concluant à un berceau africain sont imputables à Ehret. Il propose le regroupement du Sémitique, de l'Égyptien et du Berbère en Boreafrasien (Ehret 1995), qu'il date par glottochronologie à 7000 ans, alors que Couchitique et Omotique sont datés à 9000 ans. Il conforte cette « ancestralité » de l'Omotique par l'absence de référents non équivoques à des activités néolithiques dans le lexique reconstruit, alors que les branches boreafrasiennes possèdent de tels référents (Ehret 1995). Cet argument de paléolinguistique, selon Ehret, suggère à la fois que le Proto-Afro-Asiatique est pré-néolithique et, en accord avec les datations proposées, qu'il est apparu en Afrique. Il date le Proto-Afro-Asiatique à au moins 15000 ans (Ehret 1979), date pour le moins en contradiction avec la proposition de Greenberg (1964) de 9000 ans et de Diakonoff (1965) de 8000-10 000 ans. Selon 
Ehret, l'expansion de la famille aurait eu pour catalyseur l'instauration d'une économie fourragère chez les premiers groupes locuteurs de l'Afro-Asiatique en Afrique du nord-est. Le développement de l'agriculture dans cette région aurait été postérieur, vers 11 000-10 000 ans avant le présent, supposant une invention indépendante de l'agriculture en Éthiopie, à peu près contemporaine de sa naissance dans le croissant fertile.

Un certain nombre de critiques peuvent être retenues contre cet argumentaire. Certaines sont d'ordre méthodologique, d'autres ont trait au scénario évolutif dans lequel s'inscrit la naissance et l'expansion de cette famille. Un point fondamental portant flanc à la critique est la datation. Sans une chronologie précise, ayant des marges d'erreur connues et raisonnables, la séquence d'évènements ne peut être connue ni replacée dans un scénario évolutif. Les dates sur lesquelles s'appuie le scénario d'Ehret ont été obtenues par glottochronologie. Le principe de cette approche est de mesurer la date de séparation de deux langues - sachant celles-ci apparentées - en définissant un taux de remplacement du vocabulaire, et en supposant celui-ci constant sur l'ensemble du lexique de base. L'application de cette approche suppose que les langues étudiées soient génétiquement reliées, que la liste du vocabulaire de base soit standardisée, et plus fondamentalement, que la constante de remplacement du vocabulaire soit définie. La différence de 5000 à 6000 ans entre les propositions de Greenberg et de Diakonoff et celle d'Ehret pour le Proto-Afro-Asiatique s'explique par le fait que les premiers ont considéré un taux de remplacement du vocabulaire plus élevé que celui utilisé par Ehret. Toutefois, toutes deux sont postulées et non estimées à partir des données. Les listes de vocabulaire sur lesquelles cette méthode est fondée recouvrent plusieurs champs sémantiques, et il est simpliste de supposer que tous changent à la même vitesse. L'étude du remplacement lexical en fonction de la distance spatiale entre communautés locutrices a montré une non linéarité des courbes imputable à l'hétérogénéité des taux de remplacement des mots du lexique utilisé, or ces taux de remplacement dans l'espace sont fortement corrélés à ceux dans le temps (Cavalli-Sforza, Wang 1985). Par ailleurs, il s'est avéré à l'usage, en comparant les dates obtenues par cette méthode à des dates attestées par des traces écrites, que les estimations des temps de séparation ainsi calculées étaient erronées, avec des marges d'erreur excessives, et que la méthode était extrêmement sensible au choix de la liste de vocabulaire et du taux de remplacement.

La deuxième critique concerne la paléontologie linguistique. Le principe de cette méthode est de reconstruire l'environnement culturel dans lequel s'est développée une langue à partir de son lexique. Dans le cas présent, les arguments d'Ehret sont extraits $\mathrm{du}$ lexique reconstruit pour le Proto-Afro-Asiatique. Si les populations proto-afroasiatiques possédaient des mots référant à l'agriculture et à la production alimentaire, alors elles auraient pratiqué l'agriculture et auraient vraisemblablement été néolithiques. Conclure à un Proto-Afro-Asiatique pré-néolithique ne permet pas pour autant de 
trancher entre foyer africain ou asiatique. Pour Ehret, ceci est à relier directement avec la proposition d'une expansion de l'Afro-Asiatique avec l'agriculture à partir d'un foyer anatolien il y a quelque 10000 ans, selon le modèle de diffusion démique néolithique déjà proposé pour l'Indo-Européen, scénario qu'il invalide par l'absence d'une désignation d'activités aussi fondamentales que l'agriculture dans le proto-lexique. On peut toutefois questionner la représentativité du lexique reconstruit par rapport au lexique effectif, ainsi que la notion d'univocité qui permettrait d'attribuer une signification néolithique à une racine donnée. Selon Chaker (1990), un tel type d'argumentation ne serait probant que si l'on dispose «... d'un 'vocabulaire commun chamito-sémitique', sérieusement établi, sur la base d'une prise en compte systématique des données lexicales des différentes branches de la famille. On en est loin pour l'instant. »

\section{L'argumentaire extra-linguistique}

Récemment, un regain d'intérêt s'est profilé pour l'étude de l'histoire des peuplements humains dans une perspective syncrétique, à la fois biologique et culturelle. Sous l'impulsion des travaux d'Ammerman et Cavalli-Sforza (1973) en génétique des populations, relayés par Renfrew (1994) en archéologie, un modèle explicatif du changement culturel, le modèle de la diffusion démique néolithique, déjà appliqué avec succès pour l'Indo-Européen, est proposé comme également pertinent pour d'autres groupes linguistiques, et notamment pour l'Afro-Asiatique. Une analyse phylogénétique sur 42 populations mondiales et 120 systèmes alléliques (Cavalli-Sforza et al. 1994) a été fréquemment citée comme point d'ancrage de l'argumentaire sémitocentrique sur la question du foyer afro-asiatique (fig. 3). Selon cette étude, les populations afroasiatiques - au sens de locutrices de langues du groupe afro-asiatique - à savoir, une population berbère et une population sémite indéfinie du sud-ouest asiatique, sont plus proches de populations à localisation eurasiatique (Iraniens, Européens, Indiens) que de populations à localisation africaine, ce qui amène à considérer le sud-ouest asiatique comme foyer de ce groupe linguistique.

Pour conforter ce modèle, des datations au ${ }^{14} \mathrm{C}$ de sites archéologiques démontrant une activité agraire, dispersés sur le domaine actuellement occupé par l'Afro-Asiatique, ont été utilisées. Ces sites sont de plus en plus jeunes à mesure que l'on s'éloigne du croissant fertile en direction de l'Afrique de l'est, puis du nord (fig. 4).

À première vue, archéologie et génétique abondent dans le sens d'une expansion démographique des populations afro-asiatiques, liée à l'agriculture, en provenance d'un 


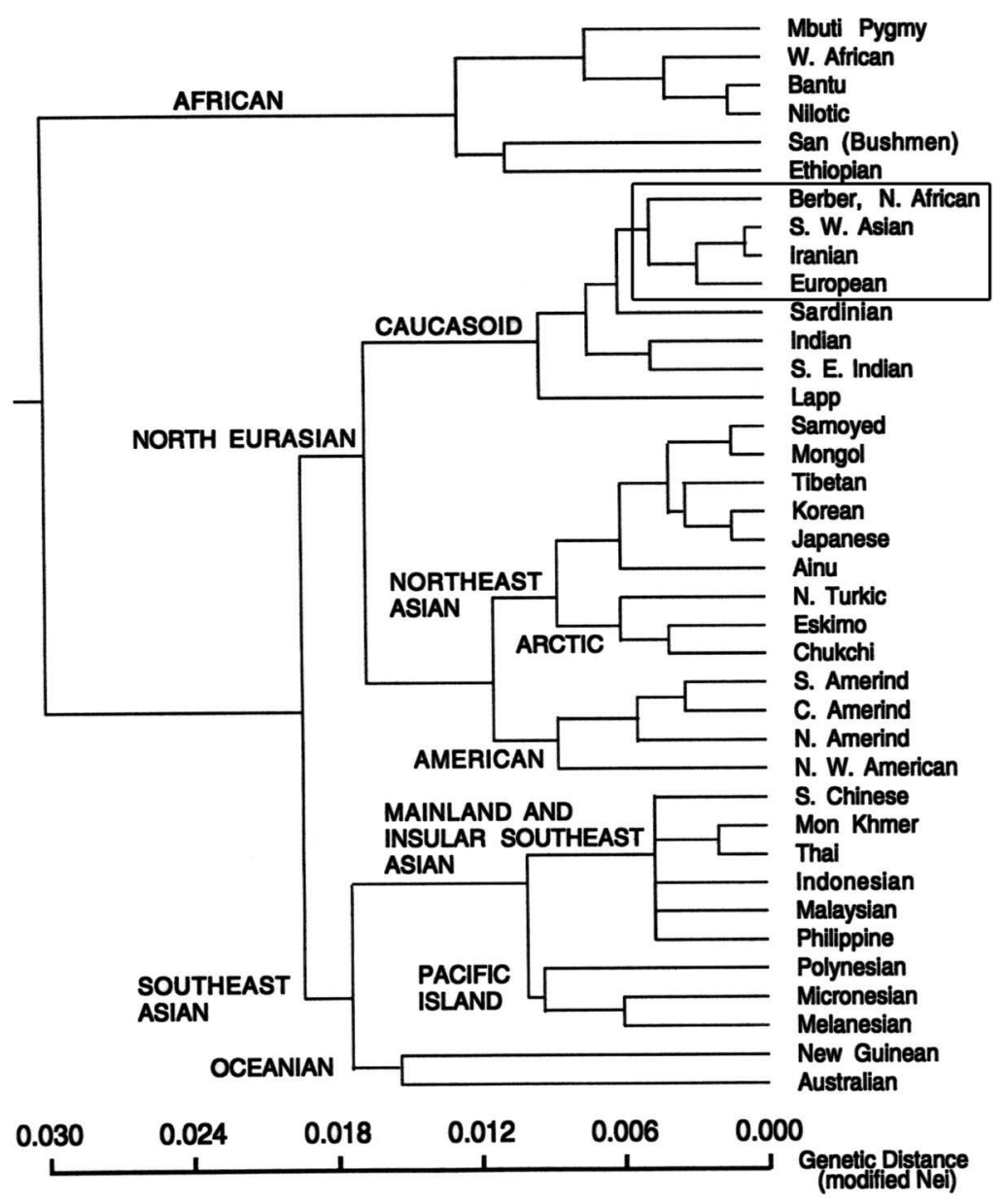

Fig. 3 - Phénogramme (distance de Nei sur 120 allèles du polymorphisme classique) de 42 populations mondiales (d'après Cavalli-Sforza et al. 1994). Les populations afro-asiatiques (encadré) sont proches de populations à localisation eurasiatique.

Fig. 3 - Phenotype chart (Nei distance on 120 genetic variants (alleles) of classic polymorphic systems) of 42 populations of the world (from Cavalli-Sforza et al. 1994). The Afro-Asiatic populations (see box) cluster with Eurasian populations. 


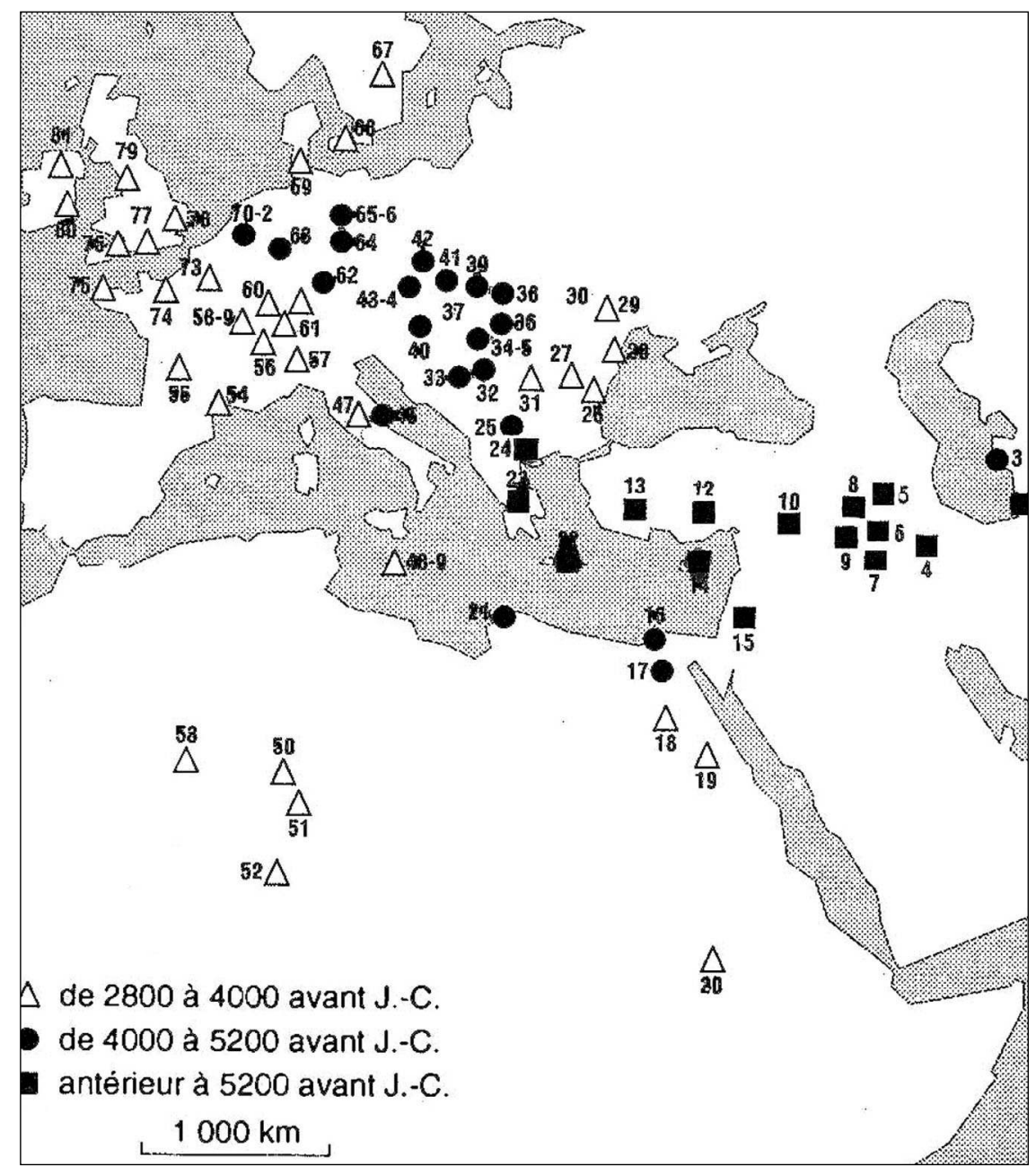

Fig. 4 - Datations au ${ }^{14} \mathrm{C}$ de sites archéologiques démontrant une activité agraire en Europe, dans le sud-ouest asiatique et en Afrique du nord et de l'est (d'après Renfrew 1994). Les sites sont plus jeunes à mesure que l'on s'éloigne de l'Asie vers l'Afrique.

Fig. 4-Radiocarbon dates for archaeological sites exhibiting agricultural activities in Europe, South-western Asia and North and East Africa (from Renfrew 1994). Sites are younger as they are further from Asia towards Africa. 
foyer anatolien et en direction de l'Afrique à travers l'Arabie. De ceci a été tirée la proposition d'une localisation du Proto-Afro-Asiatique dans le croissant fertile. Le scénario de diffusion démique sous l'impulsion de l'acquisition d'une technologie révolutionnaire comme l'agriculture est très attrayant, du fait qu'il explique les patterns de diversité génétique observés, qu'il concorde avec les datations archéologiques (début de migration il y a 10000 ans, vitesse d'expansion de $1 \mathrm{~km} / \mathrm{an}$ ) et qu'il définit le moteur des migrations. Il a d'ailleurs trouvé un écho favorable chez un courant minoritaire de la linguistique moderne, tenant d'une macro-famille linguistique, le Nostratique (Dolgopolsky 1987 ; Kaiser, Shevoroshkin 1988), regroupant les familles indo-européenne, afro-asiatique, ouralique, altaïque, dravidienne et kartvelienne (langues caucasiennes). Les branches nostratiques correspondent aux vagues de migrations majeures proposées à partir du croissant fertile anatolien, les langues se dispersant en même temps que l'agriculture par l'intermédiaire de mouvements démographiques. Ce cadre théorique détermine le berceau de l'Afro-Asiatique et le scénario d'expansion expliquant sa distribution et sa diversification actuelles. Toutefois, dans quelle mesure peut-on superposer l'expansion de l'Afro-Asiatique en tant que groupe linguistique à celle de populations parlant des langues de ce groupe ? Cette hypothèse forte doit être étayée autrement que par une interprétation synthétique des données linguistiques en fonction des données biologiques et archéologiques, d'une part parce que les méthodes de reconstruction phylogénétique utilisées pour aboutir à cette conclusion sont critiquables, d'autre part, parce que le raisonnement menace d'être circulaire : le modèle justifie la co-évolution alors même qu'il est fondé dessus. Le Nostratique reste une hypothèse de regroupement à laquelle l'Eurasiatique (Greenberg 1987) est une alternative envisageable. L'Afro-Asiatique est exclu de l'Eurasiatique dont il est groupe-frère. Dans ce cas, on peut envisager qu'il y ait découplage entre évolution génétique et évolution linguistique, au même titre que l'on peut envisager la co-évolution entre gènes et langues. Par ailleurs, l'argument basé sur l'arbre de population de Cavalli-Sforza et al. (1994) est largement interprétatif. Les Éthiopiens, bien que groupe afro-asiatique, sont plus proches des Africains que des Eurasiatiques, contrairement aux Berbères et aux Sémites du sud-ouest asiatique. Ils sont exclus de l'interprétation du fait de la grande hétérogénéité génétique observée dans cette population et de la forte influence africaine sur son patrimoine génétique actuel, qui expliquerait son comportement singulier au sein du groupe afro-asiatique. Toutefois, les Berbères ont également subi une forte contribution génétique du sud-ouest asiatique au cours des phases d'expansion islamiques, ce qui peut expliquer leur regroupement avec l'échantillon sémitique sud-ouest asiatique. Les conclusions sont antinomiques selon que l'on interprète l'arbre en tenant compte de la population éthiopienne ou de la population sud-ouest asiatique. 


\section{UNE QUESTION DE MÉTHODE}

La problématique de la localisation du berceau afro-asiatique se pose donc essentiellement en termes de méthodes. Pour l'heure, les arguments proposés sont le fruit d'interprétations des observations et non d'inférences directes soumises par des méthodologies explicites et directement falsifiables. Dans un premier temps, il est nécessaire de reconsidérer les spécificités de l'Afro-Asiatique et d'en mesurer les incidences méthodologiques. En effet, il ne faut pas perdre de vue que la problématique est linguistique. En ce sens, il est nécessaire de définir le cadre théorique qui permet de faire le lien entre la question linguistique et des arguments apportés par des disciplines telles que la génétique des populations, l'anthropologie ou l'archéologie. D'autre part, il est important de s'assurer que la question du berceau de l'Afro-Asiatique n'est pas un problème mal posé. Le regroupement initial du Sémitique avec le Berbère et le Couchitique (qui a ensuite été subdivisé en Omotique et Tchadique) est fondé sur des ressemblances lexicales et morphologiques marquées (Greenberg 1971; Chaker 1990) mais non corroborées ou inférées par la méthode comparative classique qui garantit le statut scientifique d'une famille linguistique (Chaker 1990). Dès lors se pose la question de la spécificité de ces ressemblances et donc de la monophylie de ce groupe. Or, la question de la monophylie est fondamentale : s'il n'y a pas monophylie, la problématique de la localisation du berceau ne se pose pas. La proposition alternative est que l'Afro-Asiatique est un regroupement géographique. Historiquement, d'ailleurs, la parenté chamito-sémitique a d'abord été fondée sur des considérations géographiques, anthropologiques et ethno-historiques (Chaker 1990).

\section{Monophylie de l'Afro-Asiatique}

La question de la monophylie de ce groupe, bien que fondamentale, ne peut être étudiée par des méthodes de linguistique historique d'efficacité attestée. En effet, bien que la profondeur temporelle limite à laquelle la méthode comparative de la linguistique historique - seul garant de la monophylie d'un regroupement de langues - est efficace soit discutée, elle se situe bien en deçà de la profondeur temporelle à laquelle se font les connections profondes entre les branches de l'Afro-Asiatique. Les déséquilibres dans la connaissance des évolutions des différentes branches sont extrêmes : le Sémitique est largement documenté et étudié, mais il y a peu - sinon aucune - profondeur historique pour le Berbère et le Couchitique. "Pourtant, la parenté chamito-sémitique est une certitude, perçue d'ailleurs depuis bien longtemps »(Chaker 1990). Les arguments proposés pour justifier ce regroupement (Cohen 1968 ; Diakonoff 1988), du fait de l'inapplicabilité d'une méthodologie comparatiste rigoureuse, ne distinguent pas ce qui provient de la descendance de ce qui provient d'emprunts par contact. 
En ce qui concerne la génétique des populations, la question de la monophylie de l'Afro-Asiatique n'est pas non plus tranchée. Des travaux de Cavalli-Sforza et al. (1994) sur 49 populations africaines et 47 systèmes alléliques aboutissent à un arbre phénétique où l'Afro-Asiatique apparaît polyphylétique (fig. 5). Ces résultats sont contredits par une reconstruction effectuée sur un échantillon de 130 populations mondiales (Chen et al. 1995), où l'Afro-Asiatique apparaît clairement monophylétique (fig. 6).

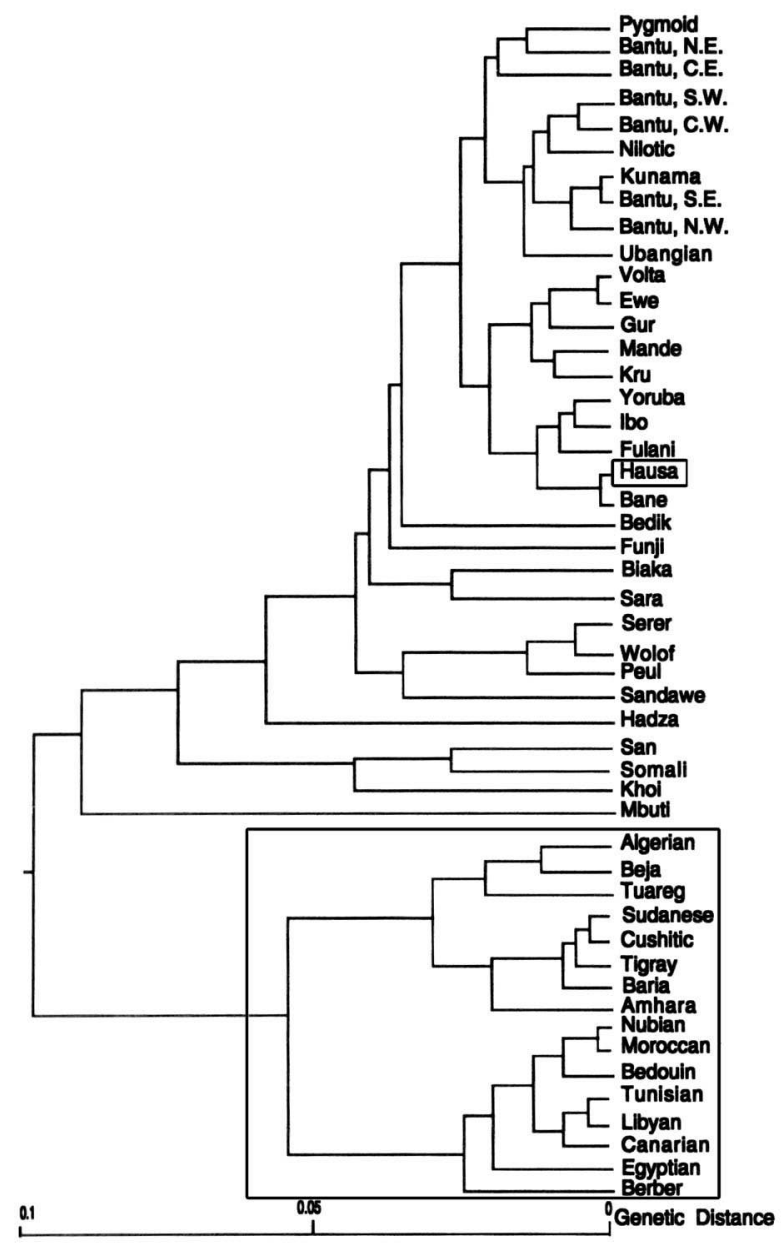

Fig. 5 - Phénogramme (distance de Nei sur allèles du polymorphisme classique) de 49 populations africaines (d'après Cavalli-Sforza et al. 1994). L'Afro-Asiatique n'apparaît pas comme un groupe monophylétique.

Fig. 5 -Phenotype chart [Nei distance on genetic variants (alleles) of classic polymorphic systems] of 49 African populations (from Cavalli-Sforza et al. 1994).

The Afro-Asiatic group is not monophyletic. 


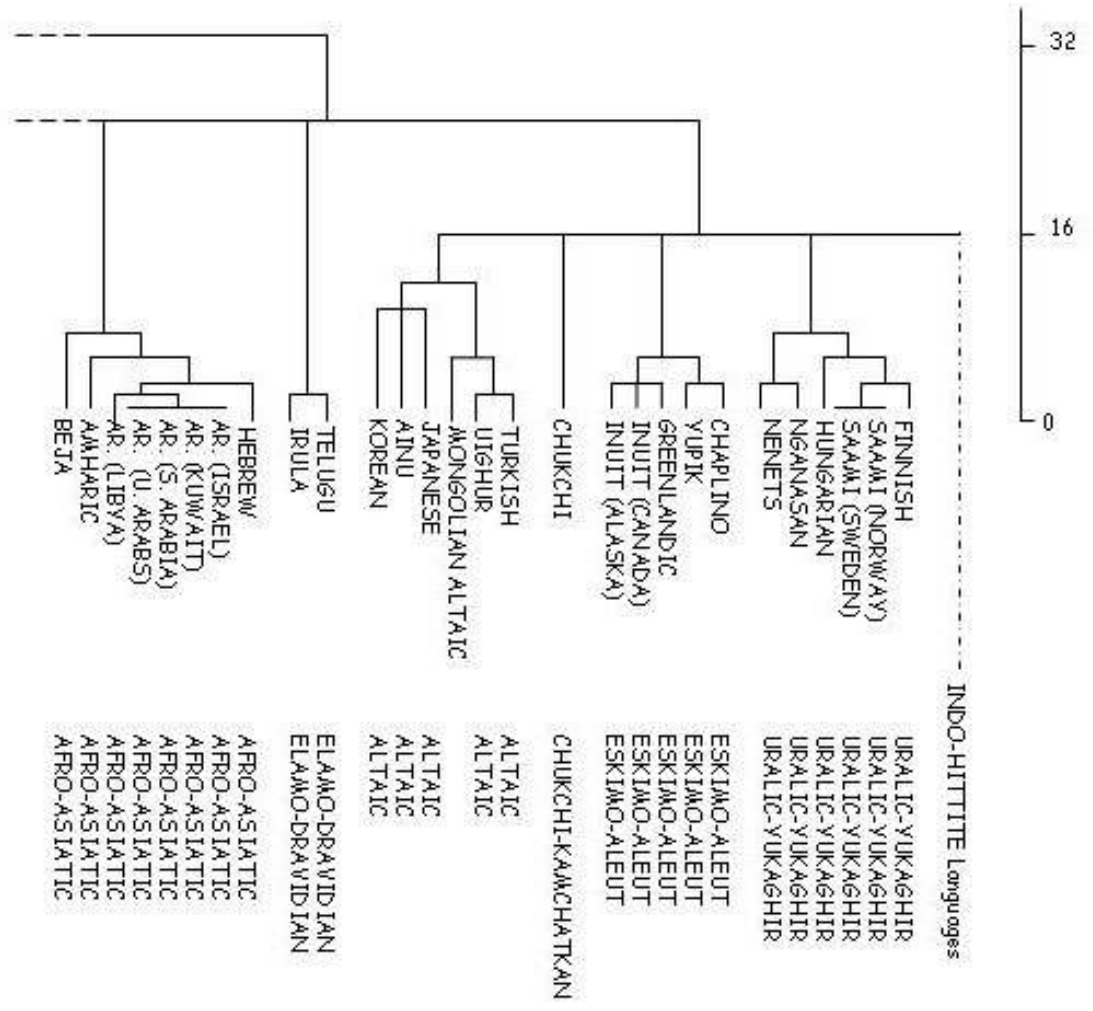

Fig. 6 - Phénogramme (distance de Prevosti sur allèles du polymorphisme classique) de 130 populations mondiales (adapté d'après Chen et al. 1995). L'Afro-Asiatique apparaît comme un groupe monophylétique.

Fig. 6 -Phenetic tree (Prevosti distance on alleles of classical polymorphic systems) of 130 populations of the world (adpated from Chen et al. 1995). The Afro-Asiatic group is monophyletic.

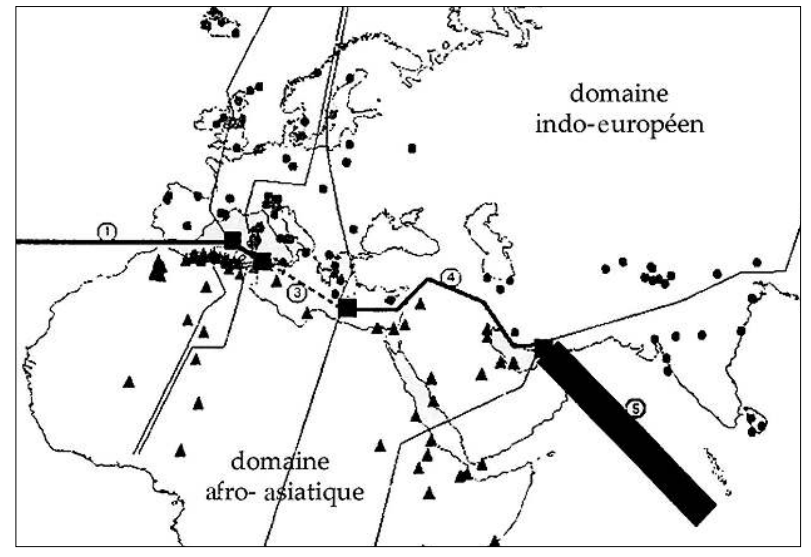

Fig. 7 - Étude de frontières entre Afro-Asiatique et Indo-Européen sur le système Rhésus (d'après Dupanloup et al. 2000). Les frontières linguistiques sont également des frontières génétiques.

Fig. 7-Analysis of frontiers between Afro-Asiatic and Indo-European on the Rhesus system (from Dupanloup et al. 2000). Linguistic frontiers are also genetic ones. 
D'autres résultats, obtenus par des méthodes d'analyse spatiale vont dans ce sens. Il s'agit d'études de frontières, telles que celles de Dupanloup et al. (2000) sur le système Rhésus, qui montrent que les frontières linguistiques entre Afro-Asiatique et Indo-Européen sont également significatives en termes de frontières génétiques (fig. 7). Toutefois, inférer la monophylie de l'Afro-Asiatique à partir de ces analyses constitue un raccourci infondé méthodologiquement.

Par ailleurs, les méthodes de reconstruction d'arbres de Cavalli-Sforza et de Chen, outre le désaccord latent entre leurs conclusions, sont des méthodes phénétiques, basées sur la ressemblance globale et non sur des hypothèses explicites de descendance. Bien que fondamentale pour aborder la problématique du berceau de l'Afro-Asiatique, ou toute étude évolutive sur ce groupe, la question de la monophylie reste largement ouverte, aussi bien d'un point de vue strictement linguistique, que d'un point de vue génétique.

\section{Questions de méthode liées aux données}

Les données biologiques dont nous disposons pour aborder ce point crucial de l'analyse sont génétiques et craniométriques. Les données génétiques sont fréquentielles et concernent des systèmes alléliques du polymorphisme classique - systèmes érythrocytaires, enzymatiques ou protéiques dont immunologiques - ou du polymorphisme non recombinant. Les données linguistiques sont essentiellement lexicales, regroupées dans la base afro-asiatique en ligne, Afro-Asiatic Index, développée à l'Oriental Institute de l'Université de Chicago par Gragg (1996). Nous envisageons également d'utiliser des données morphologiques - au sens de morphologie linguistique - accumulées sur cette famille, et invoquées dans l'argumentaire de connections de ses différentes branches. Celles-ci devront être compilées à partir de la bibliographie spécialisée.

Un certain nombre de problèmes apparaissent liés à la nature même des données. D'emblée, on constate un intérêt peu marqué pour la famille afro-asiatique du point de vue génétique. En sont symptomatiques une prospection non homogène des domaines géographique et linguistique et une mauvaise représentation des branches à détermination récente (Omotique et Tchadique). Ceci pose le problème de gestion des données manquantes. Ce problème est d'autant plus prononcé pour les marqueurs non recombinants de l'ADN mitochondrial et du chromosome Y, considérés comme plus résolutifs de questions de migrations et de relations historiques entre populations (Cavalli-Sforza 2003). Pour le contourner, nous envisageons d'utiliser la méthode du supertree (Baum 1992 ; Ragan 1992), méthode qui permet d'estimer une phylogénie à 
partir de phylogénies incomplètes reconstruites sur des ensembles de données n'échantillonnant pas les mêmes taxons.

Un autre problème apparait lié à la définition des populations. L'appartenance ethnique ou la localisation géographique sont souvent omises, et les affiliations linguistiques ne tiennent compte ni des dernières évolutions de la classification linguistique ni des éléments de l'histoire récente du domaine prospecté (exemple des populations nord-africaines, définies comme des populations arabes). Ceci pose problème dès qu'on envisage d'étudier la congruence entre arbres génétiques et arbres linguistiques, mais également lorsqu'on envisage des analyses tenant compte de la composante géographique. Cette dernière question amène à considérer de près une préoccupation majeure des linguistes quant à la représentation des connections entre langues, ou familles de langues. On constate, en ce qui concerne l'Afro-Asiatique, une certaine réticence à représenter les connections entre branches sous forme hiérarchique, sous-entendu arborée, alors même que celles-ci sont postulées comme apparentées. Cette réticence apparaît clairement dans une tendance à représenter ces connections sous la forme d'un arbre sans topologie (Cohen 1924, 1947 ; Greenberg 1955), (fig. 8).
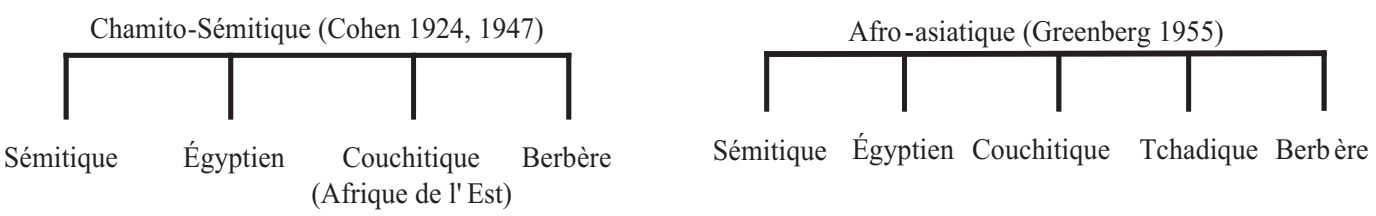

Fig. 8 - Représentations arborées non structurées de l'Afro-Asiatique.

Fig. 8 -Unstructured tree models of Afro-Asiatic.

Cette question est fondamentale : quel type d'analyse, phylogénétique ou spatiale, est le plus approprié à représenter les données ? Autrement dit, quelle représentation, arborée issue des analyses phylogénétiques classiques, ou euclidienne multidimensionnelle issue d'analyses spatiales $(A C P, M D S \ldots)$, est la plus adéquate à résumer l'information contenue dans les données. Les méthodes Qualitree et Euclide, développées par Guénoche et Garreta (2000), permettent d'évaluer l'adéquation relative de chaque type de représentation aux données, en fonction de critères métriques et topologiques. Nous envisageons d'étendre la méthode au cas particulier de la représentation géographique. Ces méthodes permettront de justifier a priori l'application 
préférentielle aux données de l'une ou l'autre des méthodes. Il faut toutefois garder à l'esprit que chaque type d'analyse apporte une part d'information différente, qu'il est intéressant de considérer pour une analyse exhaustive.

Un autre problème surgit de la nature même des données biologiques. Elles sont fréquentielles ou métriques, donc continues. Ceci impose l'utilisation de méthodes phylogénétiques capables de gérer des données continues, à savoir les méthodes phénétiques. Or, comme nous l'avons déjà mentionné, celles-ci sont fortement controversées quant à leur valeur inférentielle en termes d'affiliations génétiques. Pour résoudre ce problème particulier, non spécifique à la problématique de l'Afro-Asiatique, nous envisageons une discrétisation des données continues. Nous sommes en cours de développement d'un cadre théorique justifiant la procédure de discrétisation dans les approches phylogénétiques sur des données continues, ainsi que d'une méthode - et d'un logiciel - de discrétisation indépendants de paramètres entrés par l'utilisateur, méthode dont la validité sera testée et discutée sur des données simulées et réelles.

En ce qui concerne les données linguistiques, les limites particulières de la méthode comparative, en termes de fenêtre temporelle dans laquelle celle-ci est applicable, nous ont amené à chercher une méthode alternative qui ait démontré une égale efficacité à grande comme à faible profondeur temporelle. Notre regard s'est tout naturellement porté vers le domaine de la phylogénie, puisque l'objet de la méthode comparative est de retracer les parentés entre langues. La méthode cladistique (Hennig 1950), remplit la condition stipulée (Rosenberg et al. 2001) et présente un parallélisme méthodologique clair avec la méthode comparative (Lass 1997). De ce fait, elle nous a paru l'alternative recherchée par excellence. Toutefois, l'application de la méthode cladistique à des données lexicales implique un codage particulier. Les codages proposés dans la littérature (Gray et al. 2000 ; Holden 2002) s'appuient sur des dictionnaires de cognats établis pour les familles étudiées. Il n'en existe pas pour l'Afro-Asiatique, groupe pour lequel un codage cladistique original du lexique doit être proposé. Nous envisageons un codage sur les consonnes, celles-ci étant moins sujettes à saturation et ayant un comportement moins complexe que les voyelles, peu informatives en termes cladistiques (Ben Hamed et al., à paraître). Un tel codage nous semble approprié pour contourner le problème de l'alignement des items lexicaux lorsque ceux-ci sont polysyllabiques, problème qu'il faudra considérer par analogie à l'alignement des séquences moléculaires le cas échéant. Il permettrait également de procéder à des tests de congruence de type ILD (Farris et al. 1994), tests qui comparent des jeux de données sous une méthode unique - la parcimonie - pour évaluer la congruence sans faire implicitement référence à la structure phylogénétique. 


\section{REFORMULATION MÉTHODOLOGIQUE DE LA PROBLÉMATIQUE DE L'AFRO-ASIATIQUE}

$\mathrm{Au}$ vu de la littérature accumulée sur la question du foyer originel de l'Afro-Asiatique, nous proposons une révision méthodologique de la façon d'aborder le problème. Celle-ci se veut globale, s'appliquant indistinctement aux données rassemblées par différentes disciplines, reprenant les interrogations majeures soulevées par leurs approches spécifiques, et apportant, à défaut d'une réponse, un moyen de quantifier les hypothèses alternatives (fig. 9). Le point de départ, comme nous l'avons déjà souligné, est de tester la monophylie du groupe, aussi bien d'un point de vue linguistique que d'un point de vue génétique. Le test de monophylie se fait par rapport à des considérations géographiques et prend en compte les différentes hypothèses d'affiliations proposées pour ce groupe. La suite de l'investigation sera largement conditionnée par les résultats de ce test. S'il n'y a pas monophylie linguistique, la problématique même sera remise en cause, et il sera nécessaire de définir des groupes monophylétiques « vrais » et de reformuler la problématique d'origine et d'expansion en conséquence. Par ailleurs, si la monophylie n'est pas vérifiée pour l'un des points de vue adoptés, ceci se répercutera en termes de pertinence d'une approche pluridisciplinaire, bien que cette question soit à aborder indépendamment de la question de monophylie.

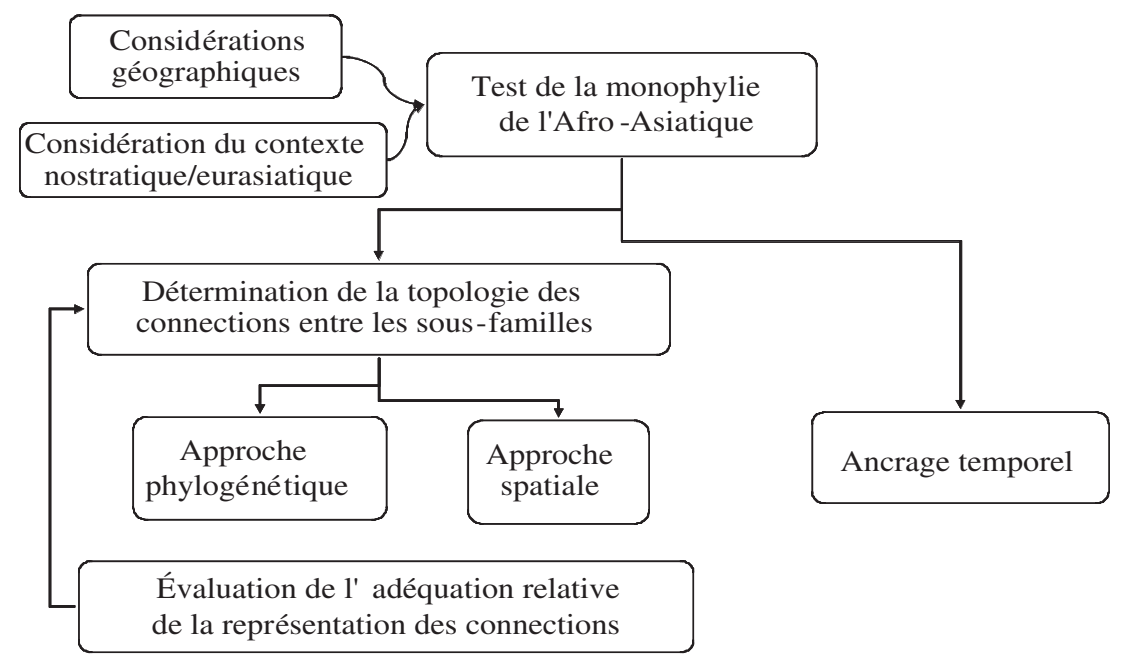

Fig. 9 - Résumé de la méthodologie proposée.

Fig. 9-Summary of the proposed methodology. 
Cette question n'est pas à envisager obligatoirement dans une perspective phylogénétique, mais peut s'appuyer sur la méthode développée par Chikhi et al. (2002), et appliquée à l'Indo-Européen, méthode qui procède d'une analyse de degré de métissage d'un ensemble de populations actuelles, par exploration d'hypothèses raisonnables sur les populations ancêtres. L'analyse sous-jacente fera écho au débat sur la représentation - et donc le mode d'analyse - des données, abordé essentiellement en linguistique. Indépendamment de cela, la question de l'ancrage temporel des reconstructions doit être envisagée.

Cette méthodologie unique sera appliquée indistinctement aux données biologiques et linguistiques. Nous disposerons donc de trois jeux de données : génétiques, anthropométriques et linguistiques, et des analyses spatiales et phylogénétiques correspondantes. Dès lors, il devient nécessaire d'envisager une synthèse des résultats obtenus en termes de coïncidence ou non-coïncidence historique. Se pose alors le problème d'évaluation de la congruence entre des histoires évolutives reconstruites à partir de jeux de données différents. La congruence est usuellement évaluée au niveau des matrices de données par des calculs de corrélations de matrices. Mais dans quelle mesure une corrélation entre matrices est-elle interprétable en termes de congruence topologique entre arbres évolutifs ? Par ailleurs, l'évaluation de la congruence topologique entre reconstruction phylogénétique n'est pas plus satisfaisante. Nous sommes en cours d'évaluation des différentes méthodes disponibles (Robinson, Foulds 1981 ; Maximum Agreement Forest...) et de constitution d'une grille permettant d'interpréter les résultats de chaque méthode d'évaluation de la congruence par rapport aux autres.

\section{CONCLUSION}

La diversité même des thèses avancées pour répondre à la problématique du foyer originel de l'Afro-Asiatique suffit à en souligner la fragilité. La question semble d'autant plus complexe qu'on l'approche d'un point de vue pluridisciplinaire. Ceci n'est pas particulier à l'Afro-Asiatique, mais émerge de toute approche syncrétique de l'histoire des peuplements humains et de la transmission culturelle, et plus généralement de la confrontation de plusieurs disciplines sur une même question. La mise en commun des connaissances acquises par chaque discipline a initialement pour objectif de simplifier la résolution d'un problème. Mais au contraire de cela, chaque méthodologie remet en question celles des autres disciplines qui en dévoilent les limites propres, apportant finalement plus de questions que de réponses. Pourtant, comme cela a déjà été souligné par Bellwood (1991), Renfrew (1992), Cavalli-Sforza (1994) et Wang (2001), entre tant 
d'autres, l'histoire des populations humaines ne trouve sa cohérence qu'en en envisageant les différentes composantes de façon synthétique. Il apparaît donc nécessaire d'accorder une attention particulière à la méthode générale de prospection de telles problématiques et de définir le cadre théorique dans lequel doit s'inscrire une approche pluridisciplinaire. Nous proposons une méthodologie unique, non spécifique d'un type particulier de données, pour éviter le piège de l'interprétation de résultats obtenus par des méthodes très différentes, aux postulats et aux contraintes particulières, et pas forcément directement comparables entre elles. Cette méthodologie unique s'accompagne d'une réflexion poussée quant aux modes de représentation des données, aux limites et aux corollaires de ceux-ci, et à la quantification de la congruence entre les résultats obtenus pour les différents jeux de données. La constitution d'une telle méthodologie fait surgir de nombreux problèmes liés à la nature même des données et à la transposition de méthodes hors de leur champ d'application usuel. Malgré cela, il parait nécessaire de persévérer dans leur résolution. Celle-ci s'accompagnera de répercussions primordiales en termes de développements méthodologiques bénéfiques aux disciplines concurrentes. Elle permettra également de définir un cadre théorique global pour une analyse syncrétique des différentes données de l'évolution des populations humaines, par une reformulation de la problématique de la congruence.

\section{Remerciements}

Nous remercions les relecteurs pour l'utile discussion qu'ils ont suscitée autour de cet article. Ce travail est effectué dans le cadre du projet «Congruence » de l'action CNRS « Origine de l'Homme, du Langage et des Langues». 


\section{BIBLIOGRAPHIE}

Ammerman (A.J.), CAVAlli-Sforza (L.L.) 1973, A population model for the diffusion of early farming in Europe, in C. Renfrew (ed.) The explanation of Culture Change, Duckworth Press, London, p. 343-357.

BAUM (B.R.) 1992, Combining trees as a way of combining datasets for phylogenetic inference, and the desirability of combining gene trees, Taxon 41: 3-10.

Bellwood (P.) 1991, The Austronesian dispersal and the origin of languages, Scientific American 265, 1: 88-93.

Ben Hamed (M.), Darlu (P.), Vallée (N.) sous presse, On cladistic reconstruction of linguistic trees through vowel data, Journal of Quantitative Linguistics.

BLENCH (R.M.) 1995, A History of Domestic Animals in Northeastern Nigeria, Cahiers de Science Humaine 31, 1: 181-238.

Cavalli-Sforza (L.L.), Feldman (M.W.) 2003, The application of molecular genetic approaches to the study of human evolution, Nature Genetics 33 suppl.: 266-275.

Cavalli-Sforza (L.L.), Menozzi (P.), Piaza (A.) 1994, The History and Geography of Human genes, Princeton University Press.

CAVAlli-Sforza (L.L.), WANG (W. S-Y) 1986, Spatial distance and lexical replacement, Language 62, 1: 38-55.

Chaker (S.) 1990, Comparatisme et reconstruction dans le domaine chamitosémitique : problème de méthodes et de limites, Travaux du Cercle Linguistique d'Aix-en-Provence 8 : 161-186.

CHEN (J.), SoKal (R.R.) 1995, Worldwide analysis of genetic and linguistic relationships of human populations, Human Biology 67, 4: 595-612.
ChiKhi (L.), Nichols (R.A.), BARbUjANi (G.), Beaumont (M.A.) 2002, Y genetic data support the Neolithic demic diffusion model, Proceedings of the National Academy of Sciences 99, 17: 11008-11013.

CoHen (D.) 1968, Les langues chamitosémitiques, Le langage, NRF-Gallimard (La Pléiade), Paris.

CoHen (M.) 1924, Les langues chamitosémitiques, in A. Meillet, M. Cohen (éds), Les Langues du monde, Librairie ancienne Édouard Champion, Paris.

CoHen (M.) 1947, Essai comparatif sur le vocabulaire et la phonétique du Chamito-Sémitique, H. Champion, Paris.

Cruciani (F.), Santolamazza (P.), Shen (P.), Macaulay (V.), Moral (P.), Olckers (A.), Modiano (D.), Holmes (S.), Destro-Bisol (G.), Coia (V.), Wallace (D.C.), Oefner (P.J.), Torroni (A.), CAVAlli-SForza (L.L.), Scozzari (R.), UNDERHILL (P.A.) 2002, A back migration from Asia to sub-Saharan Africa is supported by high-resolution analysis of human Y-chromosome haplotypes, American Journal of Human Genetics 70, 5: 1197-1214.

Diakonoff (I.M.) 1965, Semito-Hamitic Languages, Réédition révisée, 1988, Afrasian Languages, Nauka, Moscou.

Dolgopolsky (A.) 1987, The Indo-European homeland and lexical contacts of ProtoIndo-European with other languages, Mediterranean Archaeological Review 3: 7-31.

Dupanloup de Ceuninck (I.), Schneider (S.), LANGaney (A.), Excoffier (L.) 2000, Inferring the impact of linguistic boundaries on population differentiation: 
application to the Afro-Asiatic-IndoEuropean case, European Journal of Human Genetics 8, 10: 750-756.

EHRET (C.) 1979, On the antiquity of agriculture in Ethiopia, Journal of African History 20: 161-117.

EHRet (C.) 1995, Reconstructing ProtoAfroasiatic (Proto-Afrasian): Vowels, Tone, Consonants, and Vocabulary, University of California Publications in Linguistics 126, University of California Press, Berkeley and Los Angeles.

Farris (J.S.), Kallersjo (M.), Kluge (A.G.), Bult (C.) 1994, Testing significance of incongruence, Cladistics 10: 315-319.

GraGG (G.) 1996, Etymology and Electronics: The Afroasiatic Index, Oriental Institute News and Notes (Spring, 1996): $1-5$

GRAY (R.D.), JORDAN (F.M.) 2000, Language trees support the express-train sequence of Austronesian expansion, Nature 405, 6790: 1052-1055.

Greenberg (J.H.) 1955, Studies in African linguistic classification, The Hague, Mouton.

GreEnBERG (J.H.) 1964, Historical Inferences from Linguistic Research in "Sub-Saharan Africa", Boston University Papers in African History 1.

GrEENBERG (J.H.) 1971, African Languages (reprinted from Collier's Encyclopedia, 1966), in S. Anwar Dil (ed.), Language, Culture and Communication, Essays by Joseph Greenberg, Stanford University Press, Stanford.

GreEnBERG (J.H.) 1987, Language in the Americas, Stanford University Press, Stanford.
GUÉNOCHE (A.), GARRETA (H.) 2000, Quelle confiance accorder à une représentation arborée ? In Actes des Journées Biologie, Informatique et Mathématiques, AgroMontpellier, p. 181-188.

HAMmer (M.F.), KARAFET (T.), RASANAYAGAM (A.), Wood (E.T.), Altheide (T.K.), Jenkins (T.), GRIFFIths (R.C.), Templeton (A.R.), Zegura (S.L.) 1998, Out of Africa and back again: nested cladistic analysis of human Y chromosome variation, Molecular Biology and Evolution 15, 4: 427-441.

HENNIG (W.) 1950, Grundzüge einer Theorie des phylogenetischen Systematik, Deutscher Zentralverlag, Berlin, Traduction anglaise Phylogenetic Systematics, 1966.

Holden (C.J.) 2002, Bantu language trees reflect the spread of farming across subSaharan Africa: a maximum-parsimony analysis, Proceedings of the Royal Society of London series B, 269: 793-799.

Kaiser (M.), Shevoroshkin (V.) 1988, Nostratic. Annual Review of Anthropology 17: 309-329.

LASS (R.) 1997, Historical Linguistics and Language Change, Cambridge Studies in Linguistics, Cambridge University Press.

Militarev (A.), Shnirelman (V.) 1992, The Problem of a Proto-Afrasian homeland and culture (An essay in linguoarchaeological reconstruction), A Journal of Composition Theory 7: 121-130.

Passarino (G.), Semino (O.), QuintanaMurci (L.), EXCOFFIER (L.), Hammer (M.), Santachiara-Benerecetti (A.S.) 1998, Different genetic components in the Ethiopian population, identified by mtDNA and Y-chromosome polymorphismes, American Journal of Human Genetics 62, 2: 420-434. 

POUR UNE APPROCHE PLURIDISCIPLINAIRE

RaGAN (M.A.) 1992, Phylogenetic inference based on matrix representation of trees, Molecular Phylogenetics and Evolution 1: 53-58.

RENFREW (C.) 1992, Archaeology, genetics and linguistic diversity, Man 27: 445-478.

RENFREW (C.) 1994, L'énigme IndoEuropéenne : archéologie et langage, Flammarion, Paris.

Robinson (D.R.), Foulds (L.R.) 1981, Comparison of phylogenetic trees, Mathematical Biosciences 53: 131-147.

Rosenberg (M.S.), Kumar (S.) 2001, Traditional phylogenetic reconstruction methods reconstruct shallow and deep evolutionary relationships equally well, Molecular Biology and Evolution 18, 9: 1823-1827.
SAPIR (E.) 1916, Time perspective in aboriginal American culture: A study in method, in D.G. Mandelbaum (ed.), Selected writings of Edward Sapir, University of California Press, Berkeley and Los Angeles, p. 389-467.

VICYCHL (W.) 1981, Problèmes de linguistique chamitique : morphologie et vocabulaire, Comptes rendus du Groupe linguistique d'études chamito-sémitiques XVIII-XXIII : 1973-1979 (3). 